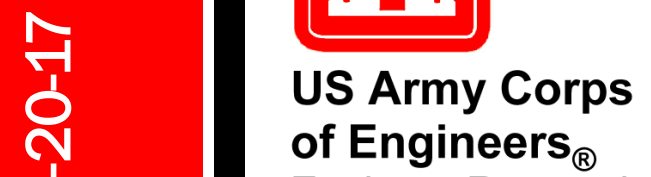

US Army Corps

Engineer Research and

Development Center

\title{
Numerical Analysis of Weak Acoustic Shocks in Aperiodic Array of Rigid Scatterers
}

Michael B. Muhlestein and Carl R. Hart

October 2020 
The U.S. Army Engineer Research and Development Center (ERDC) solves the nation's toughest engineering and environmental challenges. ERDC develops innovative solutions in civil and military engineering, geospatial sciences, water resources, and environmental sciences for the Army, the Department of Defense, civilian agencies, and our nation's public good. Find out more at www.erdc.usace.army.mil.

To search for other technical reports published by ERDC, visit the ERDC online library at https://erdclibrary.on.worldcat.org/discovery. 


\section{Numerical Analysis of Weak Acoustic Shocks in Aperiodic Array of Rigid Scatterers}

Michael B. Muhlestein and Carl R. Hart

Cold Regions Research and Engineering Laboratory

U.S. Army Engineer Research and Development Center

72 Lyme Road

Hanover, NH 03755

Final report

Approved for public release; distribution is unlimited.

Prepared for US Army Corps of Engineers
Washington, DC 20314

Under Program Element Number 611102, Project Number AB2 


\section{Preface}

This study was conducted for the US Army Corps of Engineers under Program Element Number 611102, Project Number AB2, AMSCO Code 611102AB200. The technical monitor was Mr. Ritchie L. Rodebaugh, Technical Director for Geospatial Research and Engineering.

The work was performed by the Signature Physics Branch of the Research and Engineering Division, U.S. Army Engineer Research and Development Center, Cold Regions Research and Engineering Laboratory (ERDCCRREL). At the time of publication, Dr. M. Andrew Niccolai was Branch Chief and Mr. J.D. Horne was Division Chief. The Deputy Director of ERDC-CRREL was Mr. David B. Ringelberg, and the Director was Dr. Joseph L. Corriveau.

This paper was originally published as a proceeding of the $25^{\text {th }}$ International Congress on Sound and Vibration (ICSV25), July 8-12, 2018 in Hiroshima, Japan.

The Commander of ERDC was COL Teresa A. Schlosser and the Director was Dr. David W. Pittman. 


\title{
Numerical Analysis of Weak Acoustic Shocks in Aperiodic Array of Rigid Scatterers
}

\begin{abstract}
Nonlinear propagation of shock waves through periodic structures have the potential to exhibit interesting phenomena. Frequency content of the shock that lies within a bandgap of the periodic structure is strongly attenuated, but nonlinear frequency-frequency interactions pumps energy back into those bands. To investigate the relative importance of these propagation phenomena, numerical experiments using the Khokhlov-Zabolotskaya-Kuznetsov (KZK) equation are carried out. Two-dimensional propagation through a periodic array of rectangular waveguides is per-formed by iteratively using the output of one waveguide as the input for the next waveguide. Comparison of the evolution of the initial shock wave for both the linear and nonlinear cases is presented.
\end{abstract}

\section{Introduction}

Linear wave propagation in periodic media has been well studied, usually in the context of BlochFloquet theory. However, this theory, which reduces the domain of interest from all space to a much more tractable single unit cell with periodic boundary conditions, relies heavily on the principle of linear wave superposition and is thus inappropriate for studying nonlinear wave phenomena. Previous studies of nonlinear propagation in periodic media have focused on time harmonic signals propagating in one-dimensional waveguides [1]. Nonlinear scattering of a two-dimensional plane wave from a single semi-infinite rigid barrier was studied under a paraxial approximation using similarity theory [2]. The purpose of this paper is to provide a description of pulse propagation in a periodic twodimensional system of scattering objects based on numerical simulations. These simulations are, as with Ref. [2], based on a paraxial approximation known as the Khokhlov-Zabolotskaya-Kuznetsov (KZK) equation [3].

\section{Numerical Simulation}

\subsection{Model Equation}

A full-wave simulation of finite-amplitude waves in a large system of scattering objects is very complicated and prohibitively computationally expensive, and so an approximate model based on the KZK equation is used here. The KZK equation is a time-domain parabolic equation that accounts for linear thermoviscous absorption, diffraction, and nonlinear waveform evolution, and may be written as

$$
\frac{\partial p}{\partial z}=\frac{\beta}{\rho_{0} c_{0}^{3}} p \frac{\partial p}{\partial \tau}+\frac{\delta}{2 c_{0}^{3}} \frac{\partial^{2} p}{\partial \tau^{2}}+\frac{c_{0}}{2} \int_{-\infty}^{\tau}\left(\nabla_{\perp}^{2} p\right) d \tau^{\prime},
$$


where $p$ is the acoustic pressure, $z$ is the propagation distance, $x$ and $y$ are the coordinates perpendicular to the propagation direction, $\tau=t-z / c_{0}$ is the retarded time, $c_{0}$ is the small signal sound speed, $\rho_{0}$ is the ambient density, $\beta$ is the coefficient of nonlinearity (1.2 for air), $\delta$ is the sound diffusivity $\left(\sim 10^{-5}\right.$ for air), and $\nabla_{\perp}^{2}=\left(\partial^{2} / \partial x^{2}\right)+\left(\partial^{2} / \partial y^{2}\right)$. Physically speaking, the KZK equation states that the change in the acoustic pressure as a function of the retarded time with respect to the propagation direction (left-hand side) is a linear combination of nonlinear distortion (first right-hand side (RHS) term), thermoviscous attenuation (second RHS term), and linear diffraction (third RHS term).

Equation (1) is a popular model for analyzing sound beams for its ease of implementation and its speed of calculation, but has limitations that must be acknowledged. First, being a parabolic equation the KZK equation cannot account for back-scattered sound fields, including standing waves. This means that the KZK equation cannot describe Bloch waves, linear or nonlinear, which rely on the steady state assumption. Second, the KZK equation is a narrow-angle parabolic equation, such that the diffraction described is only reliable up to about 15 degrees from the sound beam axis. Finally, a more subtle limitation is the fact that the KZK equation does not account for local nonlinearity, such as radiation pressure. The presence of scattering objects, especially large ones relative to the size of a wavelength, inherently includes significant local nonlinear effects which we will not be able to describe or account for. While these are significant limitations, there remain interesting analyses that may be performed using the KZK equation. Notably, the leading shock near the center of the sound beam may be predicted with reasonable accuracy, and will be the focus of the present study.

In order to keep the system as simple as possible, only two-dimensional propagation will be considered, and so $\partial / \partial y \rightarrow 0$. Then, defining $P=p / p_{0}, X=x / 2 a, Z=z / z_{0}, T=\omega_{0} \tau, N=z_{0} / \bar{x}$, and $A=\alpha_{0} z_{0}$, where $\omega_{0}$ is a characteristic angular frequency, $p_{0}$ is a characteristic amplitude, $a$ is a characteristic diffraction length in the transverse direction, $z_{0}=k_{0} a^{2} / 2$ is the Rayleigh distance or the characteristic diffraction length in the propagation direction, $k_{0}=\omega_{0} / c_{0}$ is the dominant wavenumber, $\bar{x}=\rho_{0} c_{0}^{3} / \beta \omega_{0} p_{0}$ is the nonlinear distortion length, and $\alpha_{0}=\delta \omega_{0}^{2} / 2 c_{0}^{3}$ is the characteristic absorption length, the KZK equation may be written as

$$
\frac{\partial P}{\partial Z}=N P \frac{\partial P}{\partial T}+A \frac{\partial^{2} P}{\partial T^{2}}+\int_{-\infty}^{T} \frac{\partial^{2} P}{\partial X^{2}} d T^{\prime} .
$$

The interpretation of the quantity $N$ is that if $N \gg 1$ nonlinear effects dominate diffraction effects, and diffraction dominates if $N \ll 1$. Thus, $N$ represents the importance of nonlinearity relative to the importance of diffraction in wave propagation. Similarly, $A$ represents the importance of linear absorption relative to the importance of diffraction. Since this work is primarily focused on the effects of nonlinearity and diffraction, $A$ is set to $10^{-6}$ (nonzero for numerical stability) for all of the simulations presented here.

Numerical solutions of Eq. (2) may be obtained using the method of fractional steps as described in Ref. [3], which leads to the three equations

$$
\begin{aligned}
& \frac{\partial P}{\partial Z}=N P \frac{\partial P}{\partial T}, \\
& \frac{\partial P}{\partial Z}=A \frac{\partial^{2} P}{\partial T^{2}}, \\
& \frac{\partial P}{\partial Z}=\int_{-\infty}^{T} \frac{\partial^{2} P}{\partial X^{2}} d T^{\prime},
\end{aligned}
$$

which are sequentially solved for a single spatial step. In the limit that the step size is much smaller than a wavelength these three equations accurately represent Eq. (2). Equation (3) may be solved for the pressure at the position $Z+\Delta Z$ based on the pressure at the position $Z$ in the time domain as [4]

$$
P(X, Z+\Delta Z, T)=P\left(X, Z, T^{\prime}\right), \quad \text { where } \quad T^{\prime}=T+N \Delta Z P(X, Z, T) .
$$




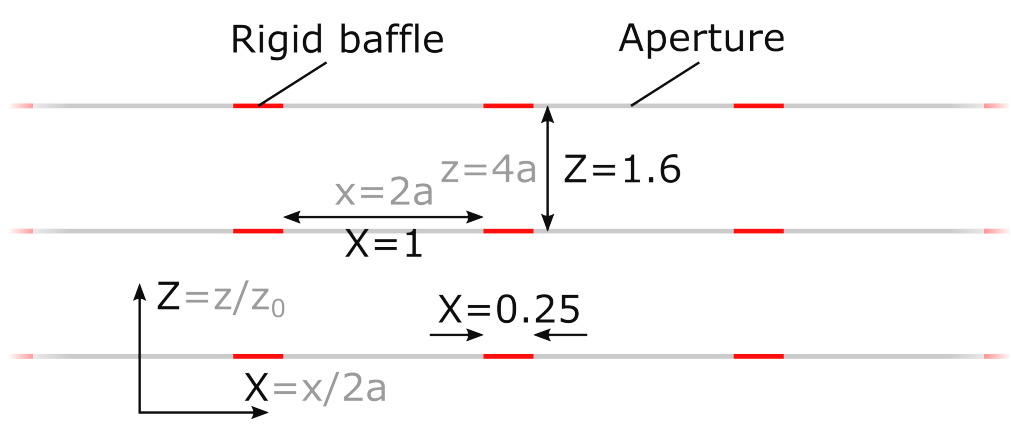

Figure 1: Schematic of the reference scattering system, which consists of an array of infinite diffraction gratings. Each diffraction grating consists of a periodic array of apertures of width $2 a$ separated by rigid baffles with a width of $a / 2$. The diffraction gratings are separated in the $z$ direction by a distance of $4 a$.

Since the $T^{\prime}$ does not match the time series $T$, linear interpolation is used to estimate this propagation step. Equations (4) and (5) are solved in the frequency domain using a Crank-Nicolson approach. Boundary conditions at the ends of the $X$ array are dictated by left and right impedances, $Z_{L}$ and $Z_{R}$, which are assumed to be infinite (rigid walls) for the present study.

\subsection{Numerical Experiment Design}

Due to the limitations of the KZK equation a complete description of scattering bodies is unnecessary. It is consistent with the approximations of the KZK equation to approximate a finite scattering body as rigid and infinitely thin (in the propagation direction) layer with a width equal to the maximum width of the scattering body. This approach means that each scatterer is effectively part of a diffraction grating, and is numerically implemented by setting the portion of the propagated pressure at all scattering boundaries equal to zero and then continuing to propagate in free space. This implementation is correct in the geometrical acoustics limit and is approximately valid for high frequency signals.

While many scattering configurations are considered below, all of them are variations on the following reference configuration. The source is chosen to be a plane wave whose time dependence is that of a Friedlander function, such that

$$
p(t, x, z=0)=p_{0} H(t)\left(1-t / t_{\mathrm{A}}\right) e^{-t / t_{\mathrm{A}}},
$$

where $H(t)$ is the Heaviside step function and $t_{\mathrm{A}}$ is the duration of the positive phase, or A-duration. It should be noted that in the results shown below the A-duration for the numerically predicted waveforms is approximated by the time it takes for the pressure to go from $5 \%$ of its absolute maximum until it first returns below 5\% of the maximum. For this waveform the dominant angular frequency is given as $\omega_{0}=2 \pi / t_{\mathrm{A}}$ and so the source may be normalized as

$$
P(T, X, Z=0)=H(T)(1-T / 2 \pi) e^{-T / 2 \pi},
$$

which has a (true) normalized A-duration of $T_{\mathrm{A}}=2 \pi$. This dominant angular frequency corresponds to a wavelength of $\lambda=c_{0} t_{\mathrm{A}}$. The scattering lattice (see Fig. 1 for a schematic) is a regular array of diffraction gratings placed parallel to the $x$ axis. Since the source is a plane wave, the dominant transverse diffracting length $a$ becomes half of an aperture width. The grating is designed such that $20 \%$ of the grating is rigid, and the aperture width $2 a$ is chosen such that $2 k_{0} a=10$. The gratings are chosen to be separated by a distance of $4 a$, which leads to a normalized separation of $4 a /\left(k_{0} a^{2} / 2\right)=$ 1.6. 


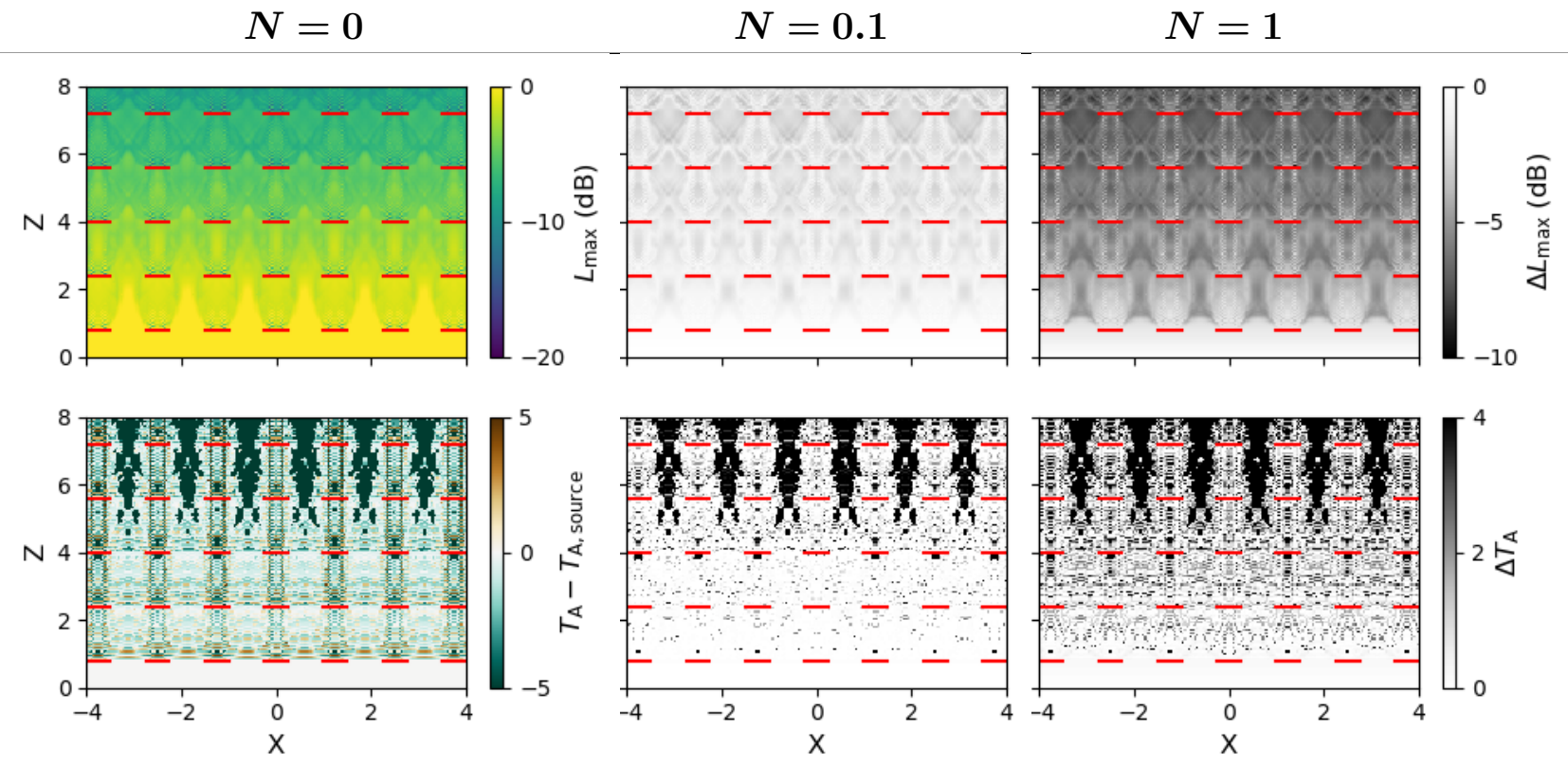

Figure 2: $(N=0)$ Calculated normalized peak pressure levels (above) and normalized A-durations (below) for the reference scattering configuration in the linear limit $(N=0)$ as a function of the normalized coordinates $X$ and $Z$. Rigid layers are denoted by red lines. ( $N=0.1$ and $N=1)$ Differences between the calculated normalized peak pressure levels (above) and normalized A-durations (below) between the nonlinear cases $(N=0.1$ and $N=1)$ and the linear $N=0$ case.

Variations on the reference configuration that are considered below include replacing the plane wave source with a baffled piston source, replacing the rectangular lattice with a triangular lattice, and replacing the infinitely thin diffraction grating with an array of finite scattering objects.

\section{Results and Analysis}

As discussed above, the limitations of the KZK equation imply that reflected waves are not captured in the calculated fields. In order to obtain useful information about the amplitude and frequency content of the initial pulse, we restrict our analysis to the normalized peak pressure level, $L_{\max }=20 \log _{10} P_{\max }$ where $P_{\max }$ is the instantaneous maximum normalized pressure, and the normalized A-duration, $T_{\mathrm{A}}$. Furthermore, the analysis should be restricted to regions away from the diffraction gratings, since the boundary condition on the backside of the gratings is only valid for geometrical acoustics and the initial acoustic field just behind the gratings depends strongly on the reflected waves. Maps of the peak pressure level and A-duration for the reference configuration with $N=0$ are shown in Fig. 2, as well as level and A-duration differences of the $N=0.1$ and $N=1$ cases relative to the $N=0$ case. The location of the rigid portions of the diffraction gratings are shown in Fig. 2 as red lines.

As may be seen in the linear case of $N=0$ shown in Fig. 2 the diffraction grating has a profound effect on $L_{\max }$ and $T_{\mathrm{A}}$. Prior to the first grating $L_{\max }$ is uniform, as is to be expected for a plane wave. After the grating $L_{\max }$ has much more structure. In the middle of the aperture $L_{\max }$ persists, but the edges gradually decrease as some of the propagated energy moves into the region behind the rigid portions of the diffraction grating or shadow zone. A similar pattern was described by Coulouvrat and Marchiano in the context of a plane wave impinging on a semi-infinte rigid screen [2]. The transition region between free space and the shadow zone is called a penumbra. Using a self-similar solution Coulouvrat and Marchiano showed that if the rigid screen exists for $X \geq 0$ and $Z=0$ then the $X$ value of the edge of the penumbra is proportional to $X^{2}$ for a single-frequency incident wave with $N=0$, and this same behavior is observed at the edges of each aperture of the first grating. While 
another series of penumbras begin at each diffraction grating, the fact that the incident waves are no longer planar complicate the successive penumbra edges with greater structure. By the third and fourth gratings the structure appears to be reaching an asymptotic condition.

The A-duration also changes with each grating. Prior to the first grating the A-duration is a constant, but after the first grating an interesting structure appears. Immediately after the first grating the A-duration briefly decreases before rising above its original value, and then drops again, rises again, etc. The amplitude of this oscillatory behavior of the A-duration decreases with distance from the grating until it roughly approaches the pre-grating value. After passing a second grating the process is renewed. After the third grating regions of very low A-duration become manifest, but the erratic nature of these regions suggests that these may be numerical artifacts. Indeed, the fine structure of the A-duration after the first two gratings suggests that the A-duration may be especially sensitive to numerical errors. Another source of error that may be contributing to these regions may be inherent limitations associated with using the KZK equation to study propagation in complicated structures.

Including nonlinearity in the propagation modifies the predicted peak pressure levels and Adurations relative to the linear case. The four right-hand plots in Fig. 2 show the difference between nonlinear and linear cases for the peak pressure level $\Delta L_{\max }$ and the A-duration $\Delta T_{\mathrm{A}}$ for the nonlinear cases of $N=0.1$ and $N=1$. The propagation distances are small enough that there is no observable difference between the linear and nonlinear cases prior to the first diffraction grating. After the first grating, however, the nonlinear cases, especially the $N=1$ case, exhibit greater peak pressure level drops than the linear case accounts for. In the $N=0.1$ case the peak pressure level decreases roughly $2 \mathrm{~dB}$ relative to the linear case, and in the $N=1$ case the peak pressure level decreases as much as $6 \mathrm{~dB}$ from the linear case. It is interesting to note that the level difference is not as great in the shadow zones. This feature results from the fact that including nonlinearity enhances the diffraction relative to the linear case [2]. The peak pressure levels for the nonlinear cases continue to decrease further relative to the linear case with each diffraction grating, but the levels in the shadow zones are more similar to the levels outside of the shadow zones. The equalization of the shadow zones is likely due to the decreasing amplitude of the incident wave (for a drop of $6 \mathrm{~dB}$ the effective value of $N$ halves), leading to more linear behavior. The A-duration is also modified by the incorporation of nonlinearity into the propagation. Again, prior to the first diffraction grating the A-duration for the nonlinear cases are practically identical to the $N=0$ case. After the first grating the A-duration becomes difficult to analyze, likely due to numerical sensitivities. However, qualitatively the A-duration appears to increase relative to the linear case with each diffraction grating for the nonlinear cases, and is enhanced by stronger nonlinearity.

Figure 3 shows level and A-duration differences for variations on the basic scattering experiment described above. For each of these experiments the nonlinearity quantity $N$ has been set equal to 0.1 and all differences are with respect to the linear case with the same configuration. The plots on the left of Fig. 3 present the results for a piston source with radius $a$ replacing the plane wave source. The middle plots show the results for a triangular lattice of apertures instead of the rectangular lattice used elsewhere. The right-hand plots show the results for a system of finite scattering bodies replacing the diffraction gratings. While each of these cases provides unique diffraction patterns and vary quantitatively from the reference case described above, the qualitative description of the results remain the same for all variations.

\section{Future Work}

The preliminary work presented here suggests that there is much more to learn about nonlinear propagation through periodic media. Questions that should be further investigated include: What is the cause for the A-duration sensitivity, and how can we reduce it? What are the characteristics of the impulse after the first arrival? What are the effects of multiple reflections on a weak shock wave, and is there some nonlinear Bloch wave-like phenomenon that manifests? How does incorporating 

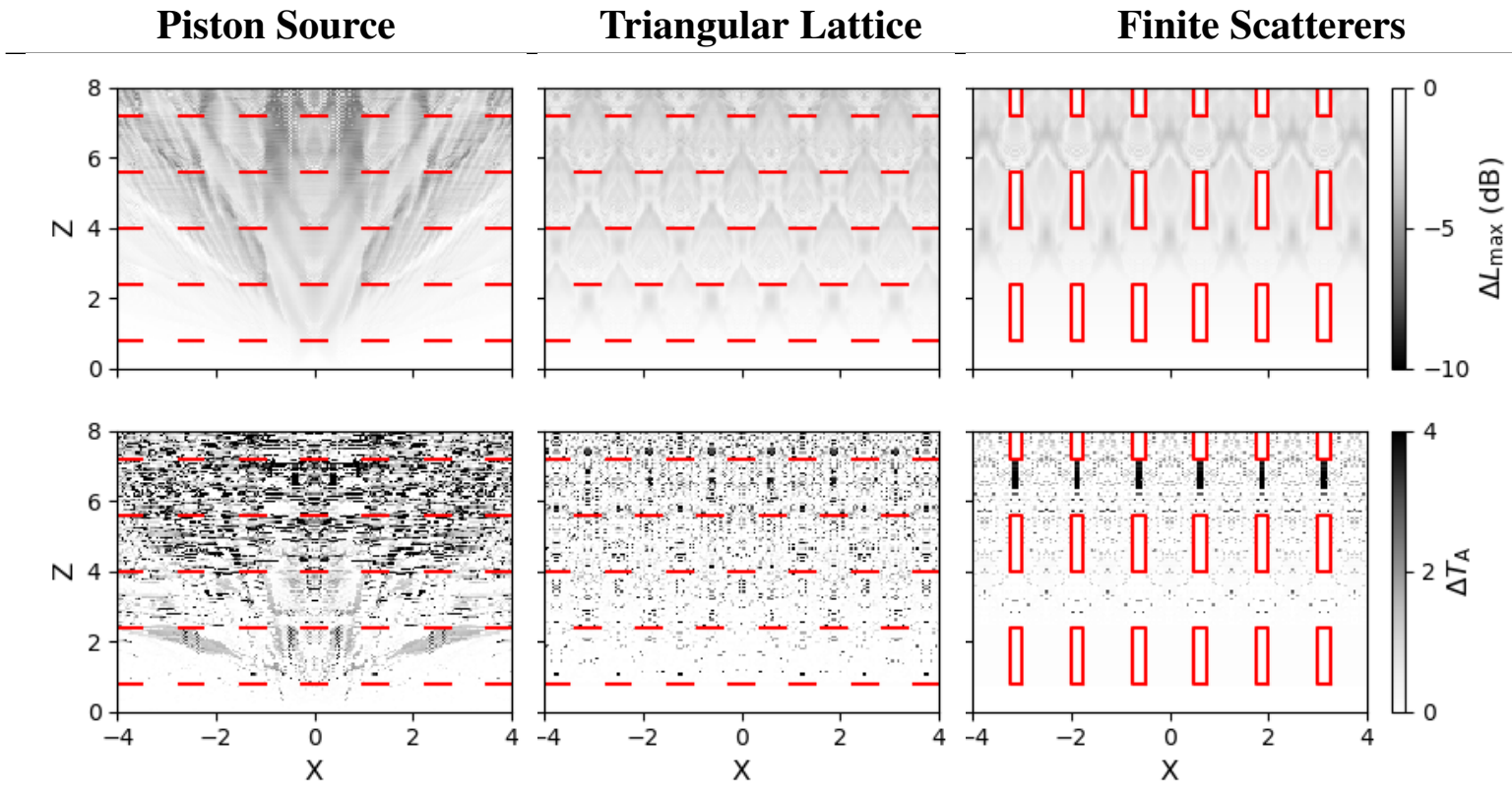

Figure 3: (Top) Peak pressure level differences (dB) and (bottom) A-duration differences of a shock propagating through a system of scatterers. (Left) A piston source is used in place of a plane wave source. (Middle) A triangular lattice of apertures is used in place of a rectangular one. (Right) finite scattering bodies replace the diffraction gratings originally u sed. In all cases $N=0.1$ and the reference case is the linear $N=0$ case.

How does incorporating wide-angle propagation modify the picture presented here?

While a full-wave simulation may be necessary to fully answer these questions, some of them may be partially answered within the context of the paraxial approximation. For example, an improved method for obtaining the A-durations may be developed. Wide angle diffraction operators designed to extend the paraxial approximation to greater angles exist which may be incorporated into the KZK equation [5]. Finally, algorithms have been developed in the context of linear acoustics which provide an estimate for two-way propagation under the paraxial approximation (see, e.g., Ref. [6]).

As with any theoretical analysis it is important to experimentally verify the results. The authors are presently in the process of developing a Schlieren system to measure the evolution of a shock wave through a periodic system of scatterers. Results from this measurement system may be used to verify and suggest improvements to the present theoretical and numerical analysis.

\section{References}

1. Bradley, C. E. Time-harmonic acoustic Bloch wave propagation in periodic waveguides. Part III. Nonlinear effects, The Journal of the Acoustical Society of America, 98 (5), 2735-2744, (1995).

2. Coulouvrat, F. and Marchiano, R. Nonlinear Fresnel diffraction of weak shock waves, The Journal of the Acoustical Society of America, 114 (4), 1749-1757, (2003).

3. Ginsberg, J. H. and Hamilton, M. F., (2008), Computational Methods. Hamilton, M. F. and Blackstock, D. T. (Eds.), Nonlinear Acoustics, pp. 309-341, Acoustical Society of America.

4. Blackstock, D. T., Hamilton, M. F. and Pierce, A. D., (2008), Progressive Waves in Lossless and Lossy Fluids. Hamilton, M. F. and Blackstock, D. T. (Eds.), Nonlinear Acoustics, pp. 65-150, Acoustical Society of America.

5. West, M., Gilbert, K. and Sack, R. A. A tutorial on the parabolic equation (PE) model used for long range sound propagation in the atmosphere, Applied Acoustics, 37 (1), 31-49, (1992). 
6. Collins, M. D. and Evans, R. B. A two-way parabolic equation for acoustic backscattering in the ocean, The Journal of the Acoustical Society of America, 91 (3), 1357-1368, (1992). 


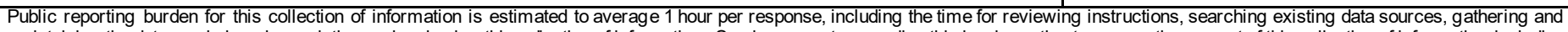

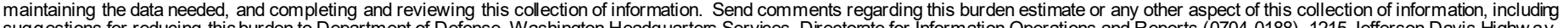

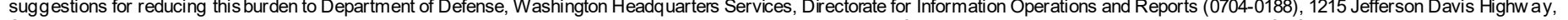

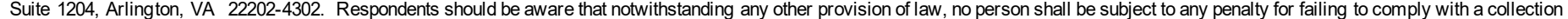

of information if it does not display a currently valid OMB control number. PLEASE DO NOT RETURN YOUR FORM TO THE ABOVE ADDRESS.

\begin{tabular}{l|l|l}
$\begin{array}{l}\text { 1. REPORT DATE } \\
\text { October } 2020\end{array}$ & $\begin{array}{c}\text { 2. REPORT TYPE } \\
\text { Final }\end{array}$ & 3. DATES COVERED (From - To)
\end{tabular}

4. TITLE AND SUBTITLE

5a. CONTRACT NUMBER

Numerical Analysis of Weak Acoustic Shocks in Aperiodic Array of

Rigid Scatterers

5b. GRANT NUMBER

5c. PROGRAM ELEMENT NUMBER

611102

6. AUTHOR(S)

5d. PROJECT NUMBER

Michael B. Muhlestein and Carl R. Hart

$\mathrm{AB} 2$

5e. TASK NUMBER

5f. WORK UNIT NUMBER

7. PERFORMING ORGANIZATION NAME(S) AND ADDRESS(ES)

U.S. Army Engineer Research and Development Center

8. PERFORMING ORGANIZATION REPORT NUMBER

Cold Regions Research and Engineering Laboratory

72 Lyme Road

ERDC/CRREL MP-20-17

Hanover, NH 03755

9. SPONSORING / MONITORING AGENCY NAME(S) AND ADDRESS(ES)

US Army Corps of Engineers

103 Pentagon

Washington, DC 20314-1000

10. SPONSOR/MONITOR'S ACRONYM(S)

11. SPONSOR/MONITOR'S REPORT NUMBER(S)

\section{DISTRIBUTION / AVAILABILITY STATEMENT}

Approved for public release; distribution is unlimited.

\section{SUPPLEMENTARY NOTES}

Origina lly published in Proceedings of the 25th International Congress on Sound and Vibration (ICSV25), July 8-12, 2018 in

Hiroshima, Japan.

Funding under AMSCO Code $611102 \mathrm{AB} 200$.

\section{ABSTRACT}

Nonlinear propagation of shock waves through periodic structures have the potential to exhibit interesting phenomena. Frequency content of the shock that lies within a bandgap of the periodic structure is strongly a ttenuated, but nonlinear frequency-frequency interactions pumps energy back into those bands. To investigate the relative importance of these propagation phenomena, numerical experiments using the Khokhlov-Zabolotskaya-Kuznetsov (KZK) equation a re carried out. Two-dimensional propagation through a periodic a ray of rectangular wa veguides is performed by iteratively using the output of one waveguide a s the in put for the next wa veguide. Comparison of the evolution of the initial shock wa ve for both the linear and nonlinear ca ses is presented.

\section{SUBJECT TERMS}

Periodic, nonlinear, scattering, numerical

\begin{tabular}{|l|l|c|c|c|l|}
\hline \multicolumn{2}{|l|}{ 16. SECURITY CLASSIFICATION OF: } & $\begin{array}{l}\text { 17. LIMITATION } \\
\text { OF ABSTRACT }\end{array}$ & $\begin{array}{l}\text { 18. NUMBER } \\
\text { OF PAGES }\end{array}$ & $\begin{array}{l}\text { 19a. NAME OF RESPONSIBLE } \\
\text { PERSON }\end{array}$ \\
\cline { 1 - 2 } $\begin{array}{c}\text { a. REPORT } \\
\text { Uncla ssified }\end{array}$ & $\begin{array}{c}\text { b. ABSTRACT } \\
\text { Unclassified }\end{array}$ & $\begin{array}{c}\text { c. THIS PAGE } \\
\text { Unclassified }\end{array}$ & SAR & 10 & $\begin{array}{l}\text { 19b. TELEPHONE NUMBER (include } \\
\text { area code) }\end{array}$ \\
\hline
\end{tabular}

